\title{
A Naturally Occurring Single Amino Acid Substitution in Human TRIM5 $\alpha$ Linker Region Affects Its Anti-HIV Type 1 Activity and Susceptibility to HIV Type 1 Infection
}

\author{
Emi E. Nakayama, ${ }^{1, *}$ Toshiaki Nakajima, ${ }^{2,3, *}$ Gurvinder Kaur, ${ }^{4}$ Jun-ich Mimaya, \\ Hiroshi Terunuma, ${ }^{6}$ Narinder Mehra, ${ }^{4}$ Akinori Kimura, ${ }^{2,3}$ and Tatsuo Shioda ${ }^{1}$
}

\begin{abstract}
TRIM5 $\alpha$ is a factor contributing to intracellular defense mechanisms against retrovirus infection. Rhesus and cynomolgus monkey TRIM5 $\alpha$ s potently restrict HIV-1, whereas human TRIM5 $\alpha$ shows weak effects against HIV-1. We investigated the association between a single nucleotide polymorphism in the TRIM5 $\alpha$ linker 2 region (rs11038628), which substituted aspartic acid (D) for glycine (G) at position 249, with susceptibility to HIV-1 infection in Japanese and Indian subjects. rs11038628 is rare in Europeans but common in Asians and Africans. Functional analyses were performed by multiple-round replication and single-round assays, and indicated that the G249D substitution attenuated anti-HIV-1 activity of human TRIM5 $\alpha$. A slight attenuation of anti-HIV-2 activity was also observed in TRIM5 $\alpha$ with 249D. The predicted secondary structure of the linker region suggested that the 249D substitution extended the $\alpha$-helix in the neighboring coiled-coil domain, suggesting that human TRIM5 $\alpha$ with 249D may lose the flexibility required for optimal recognition of retroviral capsid protein. We further analyzed the frequency of G249D in Japanese (93 HIV-1-infected subjects and 279 controls) and Indians (227 HIV-1-infected subjects and 280 controls). The frequency of 249D was significantly higher among HIV-1-infected Indian subjects than in ethnicity-matched control subjects [odds ratio $(\mathrm{OR})=1.52, p=0.026$ ]. A similar weak tendency was observed in Japanese subjects, but it was not statistically significant $(\mathrm{OR}=1.19, p=0.302)$. In conclusion, $\mathrm{G} 249 \mathrm{D}$, a common variant of human TRIM5 $\alpha$ in Asians and Africans, is associated with increased susceptibility to HIV-1 infection.
\end{abstract}

\section{Introduction}

$\mathbf{T}$

RIM5 $\alpha$ FROM RHESUS MONKEYS restricts human immunodeficiency virus-1 (HIV-1) replication at the postentry, ${ }^{1}$ preintegration stage in the viral life cycle through rapid degradation of the HIV-1 core, ${ }^{2}$ whereas human TRIM5 $\alpha$ restricts HIV-1 only weakly but potently restricts N-tropic murine leukemia virus. ${ }^{3,4}$ TRIM5 $\alpha$ is a member of the tripartite motifcontaining proteins and consists of RING, B-box 2, coiled-coil, and PRYSPRY (B30.2) domains. TRIM5 $\alpha$ recognizes the multimerized capsid (CA) proteins of an incoming virus by its $\alpha$-isoform-specific PRYSPRY domain. Studies of chimeric TRIM5 $\alpha$ s have shown that the determinant of species-specific restriction against viral infection resides in the variable regions of the PRYSPRY domain. ${ }^{5-11}$
Infection by HIV-1 and progression to acquired immune deficiency syndrome (AIDS) vary among human individuals, and these phenomena are considered to be at least partially controlled by diversity in the human genome. ${ }^{12,13}$ Two common TRIM $5 \alpha$ functional polymorphisms, H43Y and R136Q, have been studied with regard to the association with HIV-1 infection. ${ }^{14-21}$ Price et al. sequenced exon 2 of the TRIM5 gene in 1,032 women enrolled in a long-term monitored Pumwani sex worker cohort, and found that women with the R136Q polymorphism were less likely to seroconvert despite heavy exposure to HIV-1 through active sex work. ${ }^{15}$ Previous studies, including ours, showed the reduced antiviral activity of the H43Y substitution, but the associations with HIV-1 infection and disease progression were inconsistent among studies. ${ }^{14,16-20}$ Javanbakht et al. reported a paradoxical

\footnotetext{
${ }^{1}$ Department of Viral Infections, Research Institute for Microbial Disease, Osaka University, Osaka, Japan.

${ }^{2}$ Department of Molecular Pathogenesis, Medical Research Institute, Tokyo Medical and Dental University, Tokyo, Japan.

${ }^{3}$ Laboratory of Genome Diversity, School of Biomedical Science, Tokyo Medical and Dental University, Tokyo, Japan.

${ }^{4}$ Department of Transplant Immunology and Immunogenetics, All India Institute of Medical Sciences, New Delhi, India.

${ }^{5}$ Division of Hematology and Oncology, Shizuoka Children's Hospital, Shizuoka, Japan.

${ }^{6}$ Biotherapy Institute of Japan, Tokyo, Japan.

*These authors contributed equally to this work.
} 
protective effect of TRIM $5 \alpha$ with $43 \mathrm{Y}$ against HIV-1 transmission in African-Americans. ${ }^{14}$ Taken together, these findings indicate that anti-HIV-1 activity of human TRIM $5 \alpha$ cannot protect humans from an HIV-1 pandemic, but may affect the rate of HIV-1 transmission.

In the present study, we investigated the association between a single nucleotide polymorphism (SNP) in the TRIM5 $\alpha$ linker 2 region (rs11038628) between coiled-coil and PRYSPRY domains with susceptibility to HIV-1 infection. This SNP substituted aspartic acid (D) for glycine (G) at position 249. We show here that this SNP is associated with increased susceptibility to HIV-1 infection.

\section{Materials and Methods}

\section{Cloning and expression of TRIM5 $\alpha$}

The generation of recombinant Sendai viruses $(\mathrm{SeVs})$ expressing human TRIM $5 \alpha$ derived from MT4 cells, rhesus monkey TRIM $5 \alpha$ derived from LLC-MK2 cells, and cynomolgus monkey TRIM5 $\alpha$ lacking the PRYSPRY domain has been previously described. ${ }^{9,22}$ All these TRIM5 $\alpha$ s carried a hemagglutinin (HA) tag (YPYDVPDYAA) at the C-terminus. The D-to-G substitution at the 249th position was introduced into MT4 TRIM5 $\alpha$ by polymerase chain reaction (PCR) sitedirected mutagenesis. The resultant PCR fragment was cloned into pSeV18 $+\mathrm{b}(+)$ as a vector. Recombinant SeVs expressing human TRIM $5 \alpha$ carrying $G$ at position 249 were recovered according to the previously described method. ${ }^{23}$ The second passages in embryonated chicken eggs were used as stock virus for all experiments.

\section{Western blotting analysis}

MT4 cells $\left(1 \times 10^{6}\right)$ infected with recombinant SeVs expressing HA-tagged TRIM5 $\alpha$ proteins were lysed in lysis buffer (50 mM Tris- $\mathrm{HCl}, \mathrm{pH} 7.5,150 \mathrm{mM} \mathrm{NaCl}, 1 \%$ Nonidet P40, $0.5 \%$ sodium deoxycholate). TRIM $5 \alpha$ proteins in the lysates were subjected to sodium dodecyl sulfate-polyacrylamide gel electrophoresis (SDS-PAGE). Proteins in the gel were then electronically transferred onto a membrane (Immobilon; Millipore, Billerica, MA). Blots were blocked and probed with anti-HA high-affinity rat monoclonal antibody (Roche, Indianapolis, IN) overnight at $4^{\circ} \mathrm{C}$. Blots were then incubated with peroxidase-conjugated anti-rat IgG (American Qualex, San Clemente, CA), and bound antibodies were visualized with a Chemilumi-One chemiluminescent kit (Nacalai Tesque, Kyoto, Japan).

\section{Viral infection}

MT4 cells $\left(1 \times 10^{6}\right)$ were infected with SeVs expressing MT4-derived human TRIM5 $\alpha$ (249D), human TRIM5 $\alpha$ (249G), rhesus monkey TRIM $5 \alpha$, or cynomolgus monkey TRIM $5 \alpha$ lacking the PRYSPRY domain [CM-TRIM5 $\alpha-\operatorname{SPRY}(-)$ ] at a multiplicity of infection (MOI) of 10 plaque-forming units (PFU) per cell and incubated at $37^{\circ} \mathrm{C}$ for $9 \mathrm{~h}$. Aliquots of $1 \times 10^{5}$ cells were then superinfected with HIV-1 NL43 or HIV-2 GH123. Each superinfection used a titer of virus corresponding to $7 \mathrm{ng}$ of p24 of NL43 or $20 \mathrm{ng}$ of p25 of GH123. Experiments were performed with triplicate samples. The culture supernatants were collected periodically and the level of p24 or p25 was measured using a RETROtek antigen ELISA kit (ZeptoMetrix, Buffalo, NY). For the single-round infection assay, hamster TK-ts13 cells were infected with SeV expressing TRIM5 $\alpha$ as described above, and superinfected with a vesicular stomatitis virus glycoprotein (VSV-G) pseudotyped HIV-1 vector expressing green fluorescence protein (GFP) under the control of the cytomegalovirus (CMV) promoter. The original HIV-1 vector was based on the BH10 strain. ${ }^{24,25}$ To construct the lentivector possessing CA of NL4-3, we replaced the EcoRI-ApaI fragment corresponding to MA and CA of the pMDLg/p.RRE packaging vector with that of NL4-3. ${ }^{26}$ In case of HIV-2, we used a canine cell line Cf2Th and VSV-G pseudotyped HIV-2 vector expressing GFP under the control of the LTR promoter. ${ }^{27}$ Two days after infection, the cells were fixed with formaldehyde, and GFP-expressing cells were counted by a flow cytometer.

\section{Human DNA subjects}

The protocol for the present study was approved by the Ethics Review Board of the Medical Research Institute of Tokyo Medical and Dental University and that of the All India Institute of Medical Science. At setup of the cohort of HIV-1infected Japanese subjects with hemophilia in 1995, all patients had been infected for longer than 10 years but were asymptomatic without any antiviral measures. Blood samples were collected from 93 well-characterized patients who were selected from the cohort after obtaining written informed consent. $^{28,29}$ Control DNA samples were prepared from Epstein-Barr virus-transformed human B cell lines established from randomly selected healthy donors $(n=279)$ and obtained from the Japan Health Sciences Foundation. DNA samples from HIV-1-infected individuals were prepared from the blood samples using a QuickGene DNA whole blood kit S (Fujifilm, Tokyo, Japan). In addition, blood DNA samples were obtained from $227 \mathrm{HIV}$-1-infected Indian subjects and 226 healthy Indian volunteers with informed consent in related hospitals with the All India Institute of Medical Sciences, New Delhi.

\section{Identification and genotyping of nucleotide variations in TRIM5 $\alpha$ exon 5}

Primer sets were designed to amplify the genomic segments covering the entire TRIM $5 \alpha$ exon 5 as follows: sense primer (5'-GATGCGGTCATGCTATGTTG-3') and antisense primer (5'-CGAATGCTGATTTATGACCATA-3'). Genomic DNA was subjected to PCR amplification followed by sequencing using a BigDye Terminator v3.1 Cycle Sequencing Kit (Applied Biosystems, Foster City, CA). Polymorphisms were identified using the Sequencher program (Gene Code Co., Ann Arbor, MI).

\section{Statistical analysis}

All statistical analyses in this study were performed using GraphPad InStat version 3.06 for Windows (GraphPad Software, San Diego, CA). Pairwise linkage disequilibrium (LD) $\left(r^{2}\right)$ was estimated using SNPAlyze version 6.0 standard (Dynacom Co., Ltd., Chiba, Japan).

\section{Prediction of the peptide secondary structure}

The Chou-Fasman methods were used to predict the secondary structure of TRIM $5 \alpha$ using GENETYX-MAC version 15 software (Genetyx Corporation, Tokyo, Japan). 


\section{Results}

Anti-HIV-1 activity of TRIM5 $\alpha$ was attenuated by G249D substitution

We previously cloned human TRIM $5 \alpha$ from the CD4positive T cell line MT4 and noted that there is a G-to-D amino acid substitution (G249D) in comparison with the reference sequence (NM_033034). ${ }^{9}$ This position is known as a polymorphic site in the human TRIM5 gene (rs11038628) located in the linker 2 region between the coiled-coil and PRYSPRY domains (Fig. 1). Initially, we speculated that this polymorphism would have no effect on antiviral activity due to its presence in the linker 2 region. Goldschmidt et al. ${ }^{18}$ reported that HeLa cells stably transduced with TRIM5 $\alpha$ with 249D did not differ in susceptibility to HIV-1 infection. However, a tendency toward higher in vitro p24 production was observed at 7 days after infection in peripheral blood mononuclear cells from white subjects with the 249D allele, although the difference was not statistically significant mainly due to the limited number of subjects with the mutant allele. ${ }^{18}$ In addition, Old World monkey TRIM $5 \alpha$, including those of African green monkey, rhesus monkey, and cynomolgus monkey, also bears $G$ at this position (Fig. 1). The HapMap project showed the 249D allele to be rare in whites (allele frequency: 0.053) but common in Japanese (allele frequency: 0.343) and African populations (allele frequency: 0.367). These findings prompted us to reevaluate the effects of this SNP on HIV-1 infection in Asians in which the frequency of G249D is higher than in whites.

To investigate the functional significance of G249D on the anti-HIV activity of TRIM $5 \alpha$, we constructed SeV containing C-terminal HA-tagged human TRIM5 $\alpha$ (249G) (Fig. 1) by site-directed mutagenesis on MT4 TRIM5 $\alpha$, which bears D at position 249. As shown in Fig. 2A, the expression level of TRIM $5 \alpha$ (249G) was comparable to that of TRIM5 $\alpha$ (249D) in recombinant SeV-infected MT4 cells.
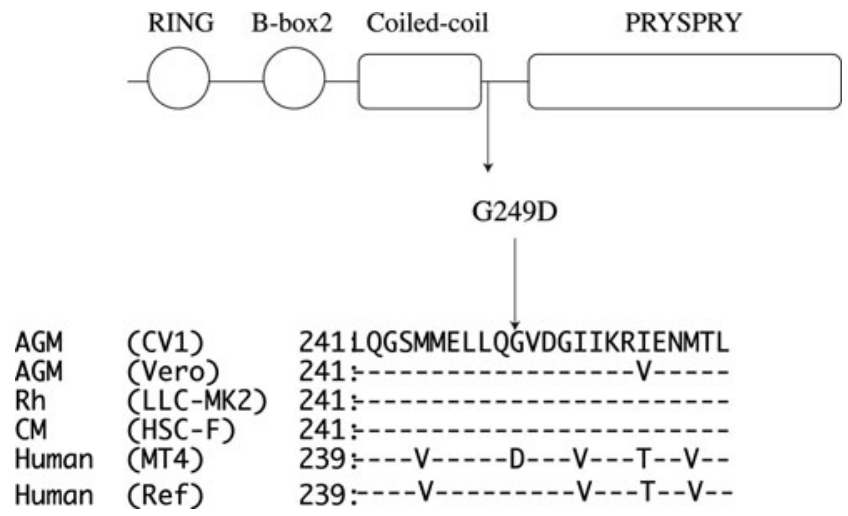

FIG. 1. Schematic presentation of TRIM $5 \alpha$ structure. Circles and squares represent functional domains of TRIM $5 \alpha$. The position of the G249D polymorphism is shown by arrows. The amino acid sequences of African green monkey (AGM) TRI$\mathrm{M} 5 \alpha$ from $\mathrm{CV}^{9}$ and Vero cells, rhesus monkey (Rh) TRIM5 $\alpha$ from LLC-MK $2,{ }^{10}$ cynomolgus monkey (CM) TRIM $5 \alpha$ from HSC-F, ${ }^{9}$ human TRIM5 $\alpha$ from MT4 cells, ${ }^{9}$ and the reference sequence (NM_033034) were aligned. Dashes denote an identical amino acid to AGM TRIM5 $\alpha$ from CV1.

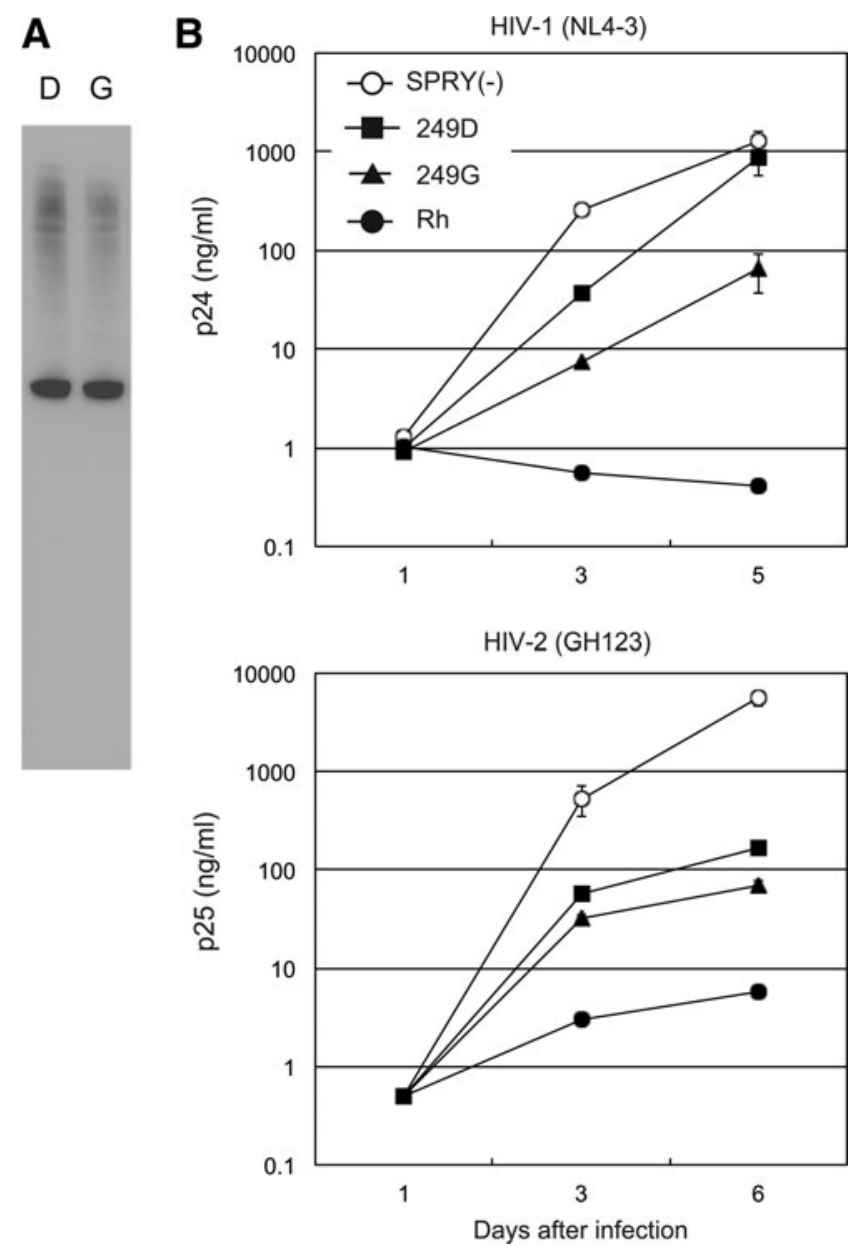

FIG. 2. (A) Lysates of MT4 cells infected with recombinant Sendai virus (SeV) expressing hemagglutinin (HA)-tagged human TRIM5 $\alpha$ with 249D (lane D) and with 249G (lane G) were visualized by western blotting with an antibody against HA. Representative results of three independent experiments are shown. (B) MT4 cells were infected with $\mathrm{SeV}$ expressing TRIM5 $\alpha$ lacking the PRYSPRY domain [SPRY (-); white circles], MT4-derived human TRIM5 $\alpha$ (249D; black squares), human TRIM5 $\alpha$ (249G; black triangles), or rhesus monkey TRIM $5 \alpha$ (Rh; black circles). Nine hours after $\mathrm{SeV}$ infection, cells were inoculated with HIV-1 strain NL4-3 or HIV-2 strain GH123, and culture supernatants were periodically assayed for levels of p24 or p25, respectively. Data points are means for triplicate samples with SD. Three and six days after infection, statistically significant differences $(p<0.05)$ of HIV-1 and HIV-2 growth were observed between human TRIM5 $\alpha$ (249D) and human TRIM5 $\alpha$ (249G) by unpaired $t$ test. Representative data of at least three independent experiments are shown.

These TRIM $5 \alpha$ constructs were tested for their ability to restrict the X4-tropic HIV-1 strain NL4-3 and HIV-2 strain GH123. MT4 cells infected with recombinant $\mathrm{SeV}$ expressing each of the TRIM $5 \alpha$ constructs were superinfected with HIV-1 NL4-3 or HIV-2 GH123. We used SeV expressing cynomolgus monkey TRIM $5 \alpha$ lacking the PRYSPRY domain as a negative control for functional TRIM $5 \alpha$, as overexpression of TRIM $5 \alpha$ lacking the PRYSPRY domain was shown to exert a dominant negative effect on endogenous human TRIM $5 \alpha .{ }^{30}$ As shown in Fig. 2B, MT4-derived human TRIM5 $\alpha$ (249D) showed only 
weak anti-HIV-1 activity, as we demonstrated previously. ${ }^{21}$ On the other hand, human TRIM5 $\alpha$ (249G) showed stronger restriction activity to HIV-1 NL4-3 than human TRIM5 $\alpha$ (249D). In the case of HIV-2, both human TRIM5 $\alpha$ with 249G and 249D exhibited apparent anti-HIV-2 activity. The human TRIM5 $\alpha$ (249G) showed stronger restriction activity to HIV-2 GH123 than human TRIM5 $\alpha$ (249D), although the difference was very small (Fig. 2B, lower panel). These results indicated that the G249D variant weakened the anti-HIV-1 and antiHIV-2 activities of human TRIM $5 \alpha$.

TRIM $5 \alpha$ is known to restrict viral infection at the early steps of HIV replication. To evaluate the anti-HIV-1 activity of human TRIM $5 \alpha$ at the early stages, we performed the singleround infection assay using a GFP expression vector (Fig. 3). The hamster cell line TK-ts13, which lacks endogenous TRI$\mathrm{M} 5 \alpha$ expression, was infected with recombinant SeV expressing human TRIM $5 \alpha$. We superinfected cells with VSV-G pseudotyped lentivector expressing GFP under the control of the CMV promoter. We used HIV-1 vectors bearing CA derived from BH10 (Fig. 3A) and NL4-3 (Fig. 3B). Both HIV-1 GFP vectors were suppressed to a greater degree by human TRIM $5 \alpha(249 G)$ than by MT4-derived human TRIM5 $\alpha$ (249D). A similar result was obtained when we used the HIV-2 GFP vector (Fig. 3C). Taken together, these observations indicated that the G249D polymorphism affected the anti-HIV-1 and anti-HIV-2 activities of human TRIM $5 \alpha$.

\section{Associations of TRIM5 $\alpha$ G249D polymorphism with susceptibility to HIV-1 infection}

We sequenced TRIM5 $\alpha$ exon 5 and found G249D in the populations tested. The associations of G249D polymorphism with susceptibility to HIV-1 infection are summarized in Table 1. The frequency of 249D was significantly higher in the HIV-1-infected Indian subjects than in the ethnicitymatched controls [odds ratio $(\mathrm{OR})=1.52, p=0.026$ ]. A similar tendency was also observed in the Japanese population, but did not reach statistical significance $(\mathrm{OR}=1.19, p=0.302)$.

Previously, we sequenced TRIM $5 \alpha$ exons 2 of the same subjects as above and reported the association of H43Y with susceptibility to HIV-1 infection. ${ }^{21}$ The levels of LD indicated that G249D in exon 5 and H43Y in exon 2 were not in tight linkage disequilibrium in either Japanese $\left(r^{2}=0.18, n=188\right)$ or Indian $\left(r^{2}=0.02, n=96\right)$ populations.

\section{Discussion}

The G249D polymorphism in TRIM5 $\alpha$ is common in Asian and African populations. It was initially speculated that there was no functional effect of this SNP, as it is located outside of any functional domains of human TRIM $5 \alpha$. Contrary to our expectation, however, we observed attenuation of anti-HIV-1 and anti-HIV-2 activity of the G-for-D substitution with both multiround replication and single-round infection assays. Furthermore, we investigated two ethnic populations, Japanese and Indian, for the G249D polymorphism and found the association of the TRIM $5 \alpha 249 \mathrm{D}$ allele with enhanced susceptibility to HIV-1 infection.

Amino acid position 249 of human TRIM5 $\alpha$ lies within the linker region for which no three-dimensional structural data have yet been reported. Therefore, we performed secondary structure prediction by the Chou-Fasman method ${ }^{31}$ to examine the possible effect of this SNP on the protein structure.
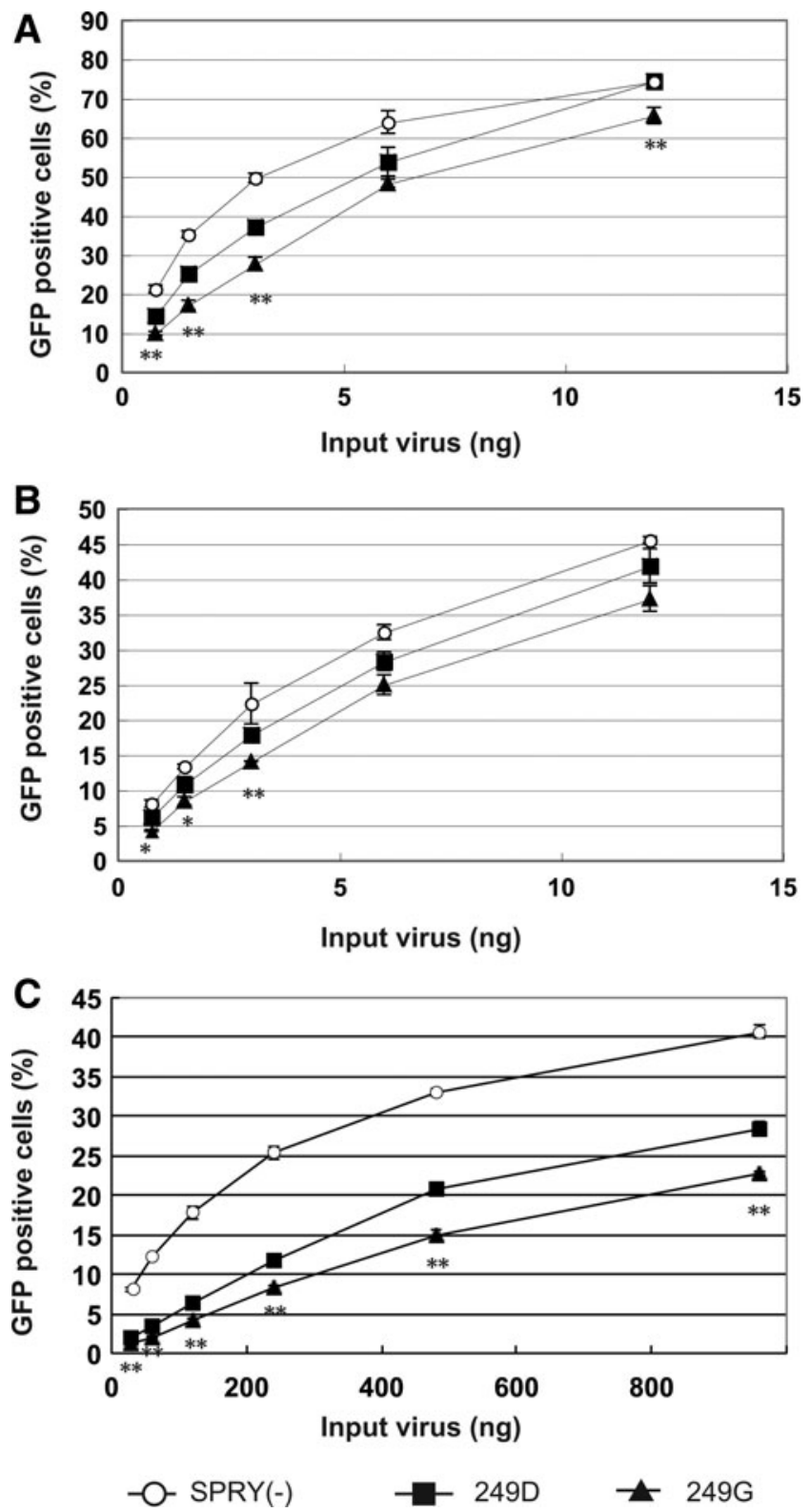

FIG. 3. TK-ts13 cells infected with SeVs expressing TRIM $5 \alpha$ lacking the PRYSPRY domain [SPRY (-); white circles], MT4derived human TRIM5 $\alpha$ (249D; black squares), or human TRIM5 $\alpha$ (249G; black triangles) were exposed to green fluorescence protein (GFP)-expressing HIV-1 vector based on BH10 (A) or NL4-3 (B). (C) Cf2Th cells infected with SeVs were exposed to an HIV-2 vector based on ROD. GFP-positive cells were counted by a flow cytometer. Data points are means for triplicate samples with SD. ${ }^{* * * *}$ The statistically significant differences, $p<0.05$ and $p<0.001$, respectively, in unpaired $t$ test between human TRIM5 $\alpha$ (249D) and human TRIM5 $\alpha$ (249G). Representative results of two independent experiments are shown.

The G-to-D substitution increased the probability of $\alpha$-helix formation and resulted in the extension of the $\alpha$-helix from the coiled-coil region into the linker 2 region. Similar results were obtained by the PREDETOR in http:// mobyle.pasteur.fr (data not shown). This suggested that TRIM $5 \alpha$ with $249 \mathrm{G}$ would be more flexible than TRIM $5 \alpha$ with 249D. 
Table 1. Association of rs10038628 (G249D) with Susceptibility to HIV-1 Infection in Japanese and Indian Populations

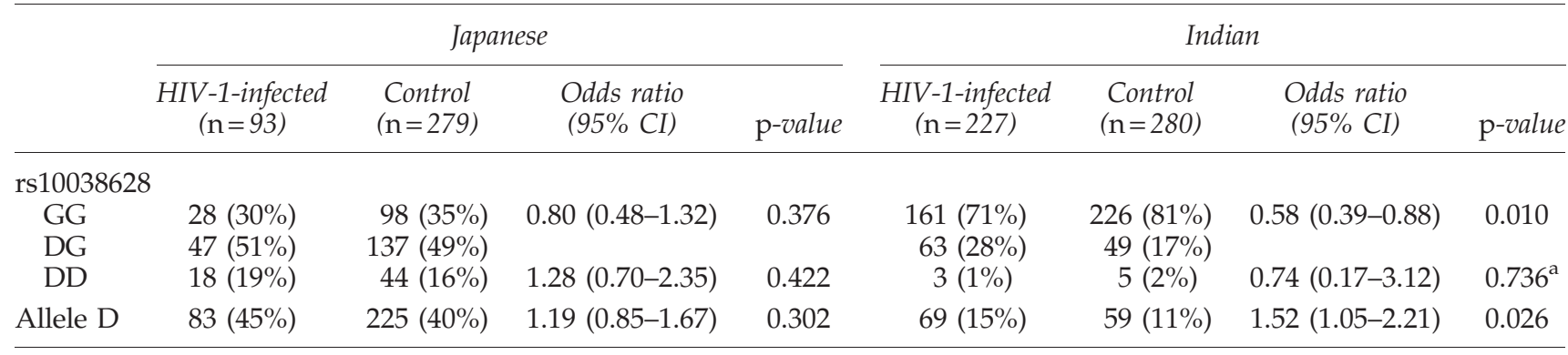

${ }^{\mathrm{a}}$ Fisher's exact test.

Human TRIM5 $\alpha$ was obviously not effective in protecting against HIV-1 infection compared with the strong Old World monkey TRIM $5 \alpha$, as only humans are susceptible and Old World monkeys are resistant to HIV-1 infection. With experimental overexpression of human TRIM $5 \alpha$, the anti-HIV-1 activity of human TRIM $5 \alpha$ was variable among previous reports. ${ }^{1,5,9,14,16,20,21}$ Our previous data showed the weakest anti-HIV-1 activity of human TRIM $5 \alpha,{ }^{9,20,21}$ even though we used the SeV system, which allowed high expression levels of inserted genes. As described in the present study, the 249D substitution would explain why our human TRIM5 $\alpha$ derived from MT4 showed little potency against HIV-1. We examined the G249D SNP in commonly used human cell lines, CEM, HeLa, Jurkat, and 293T, and found that these were all homozygous for 249G, but MT4 was homozygous for 249D. This is not surprising because the allele frequency of 249D is high in Japan but quite rare in whites and MT4 cells were established from Japanese donor blood. ${ }^{32}$ On the other hand, MT4 is highly susceptible to HIV-1 infection, ${ }^{33}$ which is in good agreement with the present data.

Previously, Goldschmidt et al. failed to observe the attenuation of antiviral activity by the 249D mutation. ${ }^{18}$ One possible reason for the discrepancy between their results and ours is the difference in expression system used. Goldschmidt et al. used HeLa cells stably transduced with TRIM5 $\alpha$ with various mutations. ${ }^{18}$ Transduced cell lines sometimes develop unexpected phenotypic changes during the cloning procedure. In contrast, we used the SeV system, and the conditions of cells infected with different recombinant viruses were always comparable, especially among those expressing full-length TRIM $5 \alpha$. It should be noted that Goldschmidt et al. also reported a tendency toward higher in vitro p24 production 7 days after infection in peripheral blood mononuclear cells from individuals with the 249D allele, which is consistent with our present results. ${ }^{18}$

We clearly showed that the 249D allele was associated with increased susceptibility to HIV-1 infection in the Indian population. However, although a similar tendency was observed in the Japanese population, the association was not significant. The precise reason why the effect of G249D was unclear in the Japanese population is not yet clear. It should be noted that our Japanese patients were infected through contaminated blood products in the early 1980s. On the other hand, the Indian patients were infected through heterosexual contact after the HIV-1 pandemic in Asia after 1990. It is possible that the difference in route of HIV-1 transmission may be responsible for this difference between Japanese and Indian patients. Further studies in well-characterized cohorts are necessary to confirm our findings regarding HIV-1 transmission and the possible effects of this SNP on AIDS progression.

\section{Acknowledgments}

We thank Ms. Setsuko Bando and Ms. Noriko Teramoto for their assistance, and Dr. Hirotaka Ode for critical suggestion. This work was supported by grants from the Ministry of Education, Culture, Sports, Science, and Technology, and the Ministry of Health, Labour, and Welfare, Japan.

\section{Author Disclosure Statement}

No competing financial interests exist.

\section{References}

1. Stremlau M, Owens CM, Perron MJ, Kiessling M, Autissier $\mathrm{P}$, and Sodroski J: The cytoplasmic body component TRIM5alpha restricts HIV-1 infection in Old World monkeys. Nature 2004;427:848-853.

2. Stremlau M, Perron M, Lee M, et al.: Specific recognition and accelerated uncoating of retroviral capsids by the TRIM5alpha restriction factor. Proc Natl Acad Sci USA 2006;103: 5514-5519.

3. Perron MJ, Stremlau M, Song B, Ulm W, Mulligan RC, and Sodroski J: TRIM5alpha mediates the postentry block to Ntropic murine leukemia viruses in human cells. Proc Natl Acad Sci USA 2004;101:11827-11832.

4. Yap MW, Nisole S, Lynch C, and Stoye JP: Trim5alpha protein restricts both HIV-1 and murine leukemia virus. Proc Natl Acad Sci USA 2004;101:10786-10791.

5. Perez-Caballero D, Hatziioannou T, Yang A, Cowan S, and Bieniasz PD: Human tripartite motif 5alpha domains responsible for retrovirus restriction activity and specificity. J Virol 2005;79:8969-8978.

6. Sawyer SL, Wu LI, Emerman M, and Malik HS: Positive selection of primate TRIM5alpha identifies a critical speciesspecific retroviral restriction domain. Proc Natl Acad Sci USA 2005;102:2832-2837.

7. Stremlau M, Perron M, Welikala S, and Sodroski J: Speciesspecific variation in the B30.2(SPRY) domain of TRIM5alpha determines the potency of human immunodeficiency virus restriction. J Virol 2005;79:3139-3145.

8. Yap MW, Nisole S, and Stoye JP: A single amino acid change in the SPRY domain of human Trim5alpha leads to HIV-1 restriction. Curr Biol 2005;15:73-78. 
9. Nakayama EE, Miyoshi H, Nagai $Y$, and Shioda T: A specific region of 37 amino acid residues in the SPRY (B30.2) domain of African green monkey TRIM5alpha determines speciesspecific restriction of simian immunodeficiency virus SIVmac infection. J Virol 2005;79:8870-8877.

10. Kono K, Song H, Shingai Y, Shioda T, and Nakayama EE: Comparison of anti-viral activity of rhesus monkey and cynomolgus monkey TRIM5alphas against human immunodeficiency virus type 2 infection. Virology 2008;373: 447-456.

11. Perron MJ, Stremlau M, and Sodroski J: Two surfaceexposed elements of the B30.2/SPRY domain as potency determinants of $\mathrm{N}$-tropic murine leukemia virus restriction by human TRIM5alpha. J Virol 2006;80:5631-5636.

12. O'Brien SJ and Nelson GW: Human genes that limit AIDS. Nat Genet 2004;36:565-574.

13. Shioda T and Nakayama EE: Human genetic polymorphisms affecting HIV-1 diseases. Int J Hematol 2006;84:12-17.

14. Javanbakht H, An P, Gold B, et al.: Effects of human TRIM5alpha polymorphisms on antiretroviral function and susceptibility to human immunodeficiency virus infection. Virology 2006;354:15-27.

15. Price $\mathrm{H}$, Lacap $\mathrm{P}$, Tuff J, et al.: A TRIM5alpha exon 2 polymorphism is associated with protection from HIV-1 infection in the Pumwani sex worker cohort. AIDS 2010;24: 1813-1821.

16. Sawyer SL, Wu LI, Akey JM, Emerman M, and Malik HS: High-frequency persistence of an impaired allele of the retroviral defense gene TRIM5alpha in humans. Curr Biol 2006;16:95-100.

17. Speelmon EC, Livingston-Rosanoff D, Li SS, et al.: Genetic association of the antiviral restriction factor TRIM5alpha with human immunodeficiency virus type 1 infection. J Virol 2006;80:2463-2471.

18. Goldschmidt V, Bleiber G, May M, Martinez R, Ortiz M, and Telenti A: Role of common human TRIM5alpha variants in HIV-1 disease progression. Retrovirology 2006;3:54.

19. van Manen D, Rits MA, Beugeling C, van Dort K, Schuitemaker $\mathrm{H}$, and Kootstra NA: The effect of Trim5 polymorphisms on the clinical course of HIV-1 infection. PLoS Pathog 2008;4:e18.

20. Nakayama EE, Carpentier W, Costagliola D, et al.: Wild type and H43Y variant of human TRIM5alpha show similar antihuman immunodeficiency virus type 1 activity both in vivo and in vitro. Immunogenetics 2007;59:511-515.

21. Nakajima T, Nakayama EE, Kaur G, et al.: Impact of novel TRIM5alpha variants, Gly110Arg and G176del, on the antiHIV-1 activity and the susceptibility to HIV-1 infection. AIDS 2009;23:2091-2100.

22. Song H, Nakayama EE, Yokoyama M, Sato H, Levy JA, and Shioda T: A single amino acid of the human immunodeficiency virus type 2 capsid affects its replication in the pres- ence of cynomolgus monkey and human TRIM5alphas. J Virol 2007;81:7280-7285.

23. Nakayama EE, Tanaka Y, Nagai Y, Iwamoto A, and Shioda T: A CCR2-V64I polymorphism affects stability of CCR2A isoform. AIDS 2004;18:729-738.

24. Miyoshi H, Takahashi M, Gage FH, and Verma IM: Stable and efficient gene transfer into the retina using an HIVbased lentiviral vector. Proc Natl Acad Sci USA 1997;94: 10319-10323.

25. Miyoshi H, Blomer U, Takahashi M, Gage FH, and Verma IM: Development of a self-inactivating lentivirus vector. J Virol 1998;72:8150-8157.

26. Kuroishi A, Bozek K, Shioda T, and Nakayama EE: A single amino acid substitution of the human immunodeficiency virus type 1 capsid protein affects viral sensitivity to TRIM5 alpha. Retrovirology 2010;7:58.

27. Miyamoto T, Nakayama EE, Yokoyama M, et al.: The carboxyl-terminus of human immunodeficiency virus type 2 circulating recombinant form 01_AB capsid protein affects sensitivity to human TRIM5alpha. PLoS One 2012;7:e47757.

28. Munkanta $M$, Terunuma $H$, Takahashi $M$, et al.: HLA-B polymorphism in Japanese HIV-1-infected long-term surviving hemophiliacs. Viral Immunol 2005;18:500-505.

29. Nakajima $T$, Ohtani $H$, Naruse $T$, et al.: Copy number variations of CCL3L1 and long-term prognosis of HIV-1 infection in asymptomatic HIV-infected Japanese with hemophilia. Immunogenetics 2007;59:793-798.

30. Maegawa H, Nakayama EE, Kuroishi A, and Shioda T: Silencing of tripartite motif protein (TRIM) 5alpha mediated anti-HIV-1 activity by truncated mutant of TRIM5alpha. J Virol Methods 2008;151:249-256.

31. Chou PY and Fasman GD: Prediction of the secondary structure of proteins from their amino acid sequence. Adv Enzymol Relat Areas Mol Biol 1978;47:45-48.

32. Akagi T, Ohtsuki Y, Shiraishi Y, and Miyoshi I: Transformation of human fetal thymus and spleen lymphocytes by human T-cell leukemia virus type I. Acta Med Okayama 1985;39:155-159.

33. Harada S, Koyanagi $\mathrm{Y}$, and Yamamoto $\mathrm{N}$ : Infection of HTLV-III/LAV in HTLV-I-carrying cells MT-2 and MT-4 and application in a plaque assay. Science 1985;229:563566 .

Address correspondence to: Tatsuo Shioda Department of Viral Infections Research Institute for Microbial Disease Osaka University

3-1, Yamada-oka, Suita-shi Osaka 565-0871 Japan

E-mail: shioda@biken.osaka-u.ac.jp 